\title{
Coherent transport in graphene nanoconstrictions
}

\author{
F. Muñoz-Rojas, D. Jacob, J. Fernández-Rossier,* and J. J. Palacios \\ Departamento de Física Aplicada, Universidad de Alicante, San Vicente del Raspeig, Spain
}

(Received 11 August 2006; published 14 November 2006)

\begin{abstract}
We study the effect of a structural nanoconstriction on the coherent transport properties of otherwise ideal zigzag-edged infinitely long graphene ribbons. The electronic structure is calculated with the standard oneorbital tight-binding model and the linear conductance is obtained using the Landauer formula. We find that, since the zero-bias current is carried in the bulk of the ribbon, this is very robust with respect to a variety of constriction geometries and edge defects. In contrast, the curve of zero-bias conductance versus gate voltage departs from the $(2 n+1) e^{2} / h$ staircase of the ideal case as soon as a single atom is removed from the sample. We also find that wedge-shaped constrictions can present nonconducting states fully localized in the constriction close to the Fermi energy. The interest of these localized states in regards to the formation of quantum dots in graphene is discussed.
\end{abstract}

DOI: 10.1103/PhysRevB.74.195417

PACS number(s): 73.23.-b, 73.63.Bd

\section{INTRODUCTION}

The recent fabrication of field effect transistor devices based both upon quasi-two-dimensional (2D) graphite quantum dots ${ }^{1}$ and upon graphene ${ }^{2-4}$ (a single atomic layer of graphite), and the observation of a new type of quantum Hall effect in the latter have triggered huge interest in the electronic properties of this system. Most of the results of standard mesoscopic physics need to be revisited ${ }^{5-7}$ in the case of graphene because its electronic structure is fundamentally different from that of metals and semiconductors where either a large density of states (DOS) at the Fermi energy or a large gap determine the properties of the materials. Graphene is a semimetal with zero DOS at the Fermi energy and zero gap. On top of that, the electronic structure close to the Fermi energy has a conical shape with perfect electron-hole symmetry, identical to that of two-dimensional massless Dirac fermions. ${ }^{8}$

Here we consider graphene-based one-dimensional flat structures, the so-called graphene nanoribbons. ${ }^{9-12}$ As opposed to two-dimensional graphene, ribbons can present a finite density of states at the Fermi energy which dominates electrical transport in undoped or weakly doped samples. Ideal graphene ribbons can be considered as the flat parent structures of carbon nanotubes, whose electronic properties have been thoroughly studied. ${ }^{14}$ Electronic transport in carbon nanotubes has been studied in different regimes, including ballistic, ${ }^{15}$ Coulomb blockade, ${ }^{16}$ and Kondo. ${ }^{17,18}$ Progress in the fabrication of graphene based nanostructures that permits one to study transport in graphene ribbons motivates this work.

The presence of edges makes the electronic structure of graphene ribbons different from that of nanotubes. Two types of idealized edges are usually considered: Armchair and zigzag. ${ }^{9}$ Interestingly, all the zigzag and some of the armchair edges result in a band at the Fermi energy. ${ }^{9}$ In the case of narrow zigzag-edged ribbons, the top and bottom edge states can be sufficiently close as to make hybridization possible, resulting in two low energy dispersive bands, symmetrically placed around the Fermi energy, $E=0$. Depending on their width, nanoribbons with armchair edges can be me- tallic or insulating. The different density of states for armchair and zigzag edges has been experimentally observed in scanning tunnel microscope (STM) experiments with atomic resolution. ${ }^{19,20}$

Coherent or quantum transport in graphene ribbons has been studied previously, both for ideal ${ }^{13}$ and defective ${ }^{21,22}$ cases in the infinite length $L$ case. The case of disorder-free graphene ribbons with finite $L$ comparable to the width $W$ has also been studied in connection with the experimental measurement of a minimum conductivity in mesoscopic size graphene layers. ${ }^{5,6}$ In the spirit of quantum point contact physics, in this work we study the coherent transport of infinitely long graphene ribbons, narrower than $W \simeq 5 \mathrm{~nm}$, with a structural nanometric constriction like the one schematically shown in Fig. 1. This type of structure can also be considered as an idealization of an all-carbon singlemolecule junction. In conventional single-molecule junctions the organic molecule is attached to metallic electrodes. Here these are replaced by perfect graphene ribbons and the role of the molecule is played by a geometrical constriction. Due to the very different electronic structure displayed by different all-carbon nanostructures ${ }^{14}$ the conduction properties of these systems do not appear obvious a priori to us.

We calculate the electronic structure in the one orbital tight binding (TB) approximation. The relevant orbital is the $p_{z}$, since the $s p^{2}$ orbitals form bonding and antibonding states

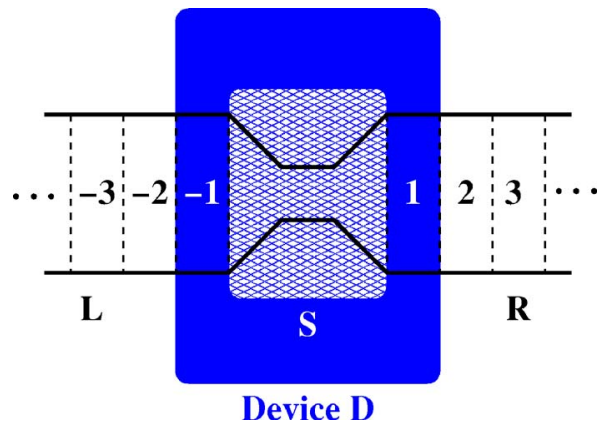

FIG. 1. (Color online) Division of system into leads L and R, device D, and scattering region S (left). Division of operator matrices into corresponding submatrices (right). 
very far away in energy. In the TB approximation ideal twodimensional graphene has a conical energy dispersion at low energies and the Fermi surface $(E=0)$ is formed by six points. This low energy region can be described in terms of a $\vec{k} \cdot \vec{p}$ theory whose mathematical structure is very similar to the Dirac theory for massless fermions, ${ }^{8}$ which yields physical insight. The $\vec{k} \cdot \vec{p}$ has also been worked out for edge states in ideal ribbons. ${ }^{12}$ The TB approach provides natural energy and momentum cutoffs to the $\vec{k} \cdot \vec{p}$ theory, permits one to model perturbations at the atomic scale, and is a good preliminary step towards $a b$ initio calculations. ${ }^{23}$

The rest of the paper is organized as follows. In Sec. II we review the transport formalism. In Sec. III we review the electronic structure of ideal ribbons and we study the effect of vacancies, located at the edge of the ribbons, on their transport properties. We find that the conductivity of undoped graphene ribbons is weakly affected by the presence of this kind of disorder. In Secs. IV and V we study transport properties of square- and wedge-shaped constrictions. Whereas the former present finite conductance, the latter have a vanishing transmission at low energies, coexisting with a finite density of localized states. These states at energies close to zero form what can be called a quantum dot in graphene. In Sec. VI we discuss the validity of our approximations and we summarize and discuss the main results of this paper.

\section{TRANSPORT THEORY}

The natural framework for transport calculations in nanoscopic devices is the Landauer formalism. The description of electron transport within the Landauer formalism is based on the assumption that transport across the highest resistance region is coherent, i.e., inelastic scattering is negligible there. A more complete account can be found, e.g., in the book by Datta. ${ }^{24}$ In what follows we will assume that the inelastic mean free path for graphene electrons is much longer than the typical dimensions of the nanoconstrictions considered and than the ribbon widths. According to recent theory work, inelastic scattering due to phonons is very inefficient in graphene ribbons so that our assumption seems to be met even at room temperature. ${ }^{25}$ This makes elastic scattering the major contributor to resistance. In contrast to metallic systems where the resistance of the electrodes is negligible compared to that of the nanoconstriction, in the case of graphene nanoconstrictions, the low-bias conductance of the ideal electrode is also very small, $\frac{2 e^{2}}{h}$. The consequences of this are explored in what follows.

We consider the effect of constrictions on the transport properties of an otherwise ideal ribbon (Fig. 1). The system has three regions: the central region (or device) where the constriction is located and the left and right leads. The latter are described as semi-infinite one-dimensional perfect ribbons of finite width, characterized by the number of atoms in the unit cell, $N$. We only consider ribbons with zigzag edges as the ones studied in previous works. ${ }^{9} 10,21$ We consider constrictions of various shapes, from the removal of a single atom or a few atoms on the edge to square-shaped and wedge-shaped constrictions.
The introduction of the constriction breaks the translational symmetry of the perfect lead so that, in general, electrons incident on a given band will be either reflected or transmitted into other bands after hitting the constriction. The square of the transmission amplitude $t_{n m}$ gives the probability of an incoming mode $m$ to be scattered on an outcoming mode $n$. The Landauer formula links the overall transmission

$$
T(E)=\sum_{m, n}\left\|t_{n m}\right\|^{2}=\operatorname{Tr}\left[t^{\dagger} t\right]
$$

with the linear conductance

$$
G\left(E_{F}\right)=\frac{2 e^{2}}{h} T\left(E_{F}\right),
$$

where $E_{F}$ is the Fermi energy.

For completeness, we review the basic steps in the calculation of the transmission $T(E)$ using single-particle Green's functions as routinely done in nanoelectronics. ${ }^{24}$ In the TB approach used here, the Hamiltonian matrix is straightforwardly obtained for a given atomic structure. The leads are characterized by a unit cell with $N$ atoms and a propagation direction which we take along the $x$ axis. When written in blocks $N \times N$, the Hamiltonian of the leads is a semi-infinite tridiagonal matrix, with intracell blocks $H_{0}$ and an intercell first-neighbor coupling $V$. Our starting point is the partition of the Hamiltonian of the infinite system in three regions. The choice of the boundaries between device and leads is done so that left and right electrodes are not directly coupled. For convenience the device region is always chosen so that the left and right boundaries are given by one of these units cells. Therefore the coupling between the surface unit cell of the left (right) electrode to the right (left) boundary of the device is given by the same intercell matrix $V$. According to this scheme, the Hamiltonian matrix set is divided into submatrices as follows:

$$
H=\left(\begin{array}{ccc}
H_{\mathrm{L}} & H_{\mathrm{LD}} & 0 \\
H_{\mathrm{DL}} & H_{\mathrm{D}} & H_{\mathrm{DR}} \\
0 & H_{\mathrm{RD}} & H_{\mathrm{R}}
\end{array}\right) .
$$

Here $H_{\mathrm{D}}$ is a finite size square matrix with range equal to the number of atoms in the device, $N_{d}$. In contrast, $H_{\mathrm{L}, \mathrm{R}}$ are infinite size square matrices describing the semi-infinite electrodes and $H_{\mathrm{DL}}$ and $H_{\mathrm{LD}}$ are infinite rectangular matrices. The Green function operator, defined as

$$
(E-H) G(E)=1
$$

can also be divided into submatrices as

$$
G=\left(\begin{array}{ccc}
G_{\mathrm{L}} & G_{\mathrm{LD}} & G_{\mathrm{LR}} \\
G_{\mathrm{DL}} & G_{\mathrm{D}} & G_{\mathrm{DR}} \\
G_{\mathrm{RL}} & G_{\mathrm{RD}} & G_{R}
\end{array}\right) .
$$

After simple steps, it is possible to write the Green function of the device as 


$$
G_{\mathrm{D}}(E)=\left[E-H_{\mathrm{D}}-\Sigma_{\mathrm{L}}(E)-\Sigma_{\mathrm{R}}(E)\right]^{-1},
$$

where $\Sigma_{\mathrm{L}, \mathrm{R}}$ are the so-called self-energy $N_{d} \times N_{d}$ matrices given by

$$
\begin{aligned}
& \Sigma_{\mathrm{L}}(E):=H_{\mathrm{DL}} g_{\mathrm{L}}(E) H_{\mathrm{LD}}, \\
& \Sigma_{\mathrm{R}}(E):=H_{\mathrm{DR}} g_{\mathrm{R}}(E) H_{\mathrm{RD}} .
\end{aligned}
$$

The self-energies $\Sigma_{\mathrm{L}}(E)$ and $\Sigma_{\mathrm{R}}(E)$ describe the effect of the electrodes on the electronic structure of the device. The real part of the self-energy results in a shift of the device levels whereas the imaginary part provides a lifetime. The device self-energies are given by the Green's functions of the semiinfinite isolated leads $g_{\mathrm{L}}(E)=\left(E-H_{\mathrm{L}}\right)^{-1}$ and $g_{\mathrm{R}}(E)=(E$ $\left.-H_{\mathrm{R}}\right)^{-1}$ projected into the device region by the coupling of the leads to the device $H_{\mathrm{DL}}$ and $H_{\mathrm{RD}}$. In contrast to the Green function of an infinite system with translational invariance, the calculation of the Green function of a semi-infinite system with a surface is nontrivial.

We can write the surface part of the semi-infinite Green function $g_{\mathrm{L}, \mathrm{R}}(E)$ of the electrode as

$$
\left.g_{\mathrm{L}, \mathrm{R}}(E)\right|_{\text {surface }}=\frac{1}{E-H_{0}-\Sigma_{1, \mathrm{r}}(E)},
$$

where $\Sigma_{1, \mathrm{r}}$ is a self-energy (different from $\Sigma_{L, R}$ ) that accounts for the effect of the rest of the semi-infinite chain on the first unit cell. In one dimension it is possible to derive a recursive relation that yields a self-consistent Dyson equation for this self-energy:

$$
\Sigma_{1, \mathrm{r}}(E)=V \frac{1}{E-H_{0}-\Sigma_{1, \mathrm{r}}(E)} V^{\dagger}
$$

The coupling matrices $\Gamma_{\mathrm{L}}(E)$ and $\Gamma_{\mathrm{R}}(E)$ are defined as the difference between the retarded and advanced self-energy of the leads projected into the device by the coupling $H_{\mathrm{DL}}$ and $H_{\mathrm{RD}}$ :

$$
\Gamma(E)_{\mathrm{L}(\mathrm{R})}=i\left(\Sigma_{\mathrm{L}(\mathrm{R})}(E)-\Sigma_{\mathrm{L}(\mathrm{R})}^{\dagger}(E)\right) .
$$

With all these ingredients, we can compute the transmission using the result ${ }^{24}$

$$
T(E)=\operatorname{Tr}\left[\Gamma_{\mathrm{L}}(E) G_{\mathrm{D}}^{\dagger}(E) \Gamma_{\mathrm{R}}(E) G_{\mathrm{D}}(E)\right] .
$$

Therefore for a given system, we first compute the electrode surface Green function (6) by solving the Dyson equation (7). This permits one to compute the device self-energy (5), the device Green function (4) and the coupling matrices (8). The final step is the calculation of the transmission function.

\section{IDEAL AND WEAKLY DEFECTIVE ZIGZAG RIBBONS}

\section{A. Ideal ribbons}

In this section we briefly review the electronic structure and transport properties of both ideal and weakly defective zigzag-edged ribbons. We consider ribbons with two different widths whose unit cells are composed of $N=24$ and $N$ $=48$ atoms, respectively. The supercell unit of the $N=24$ rib-
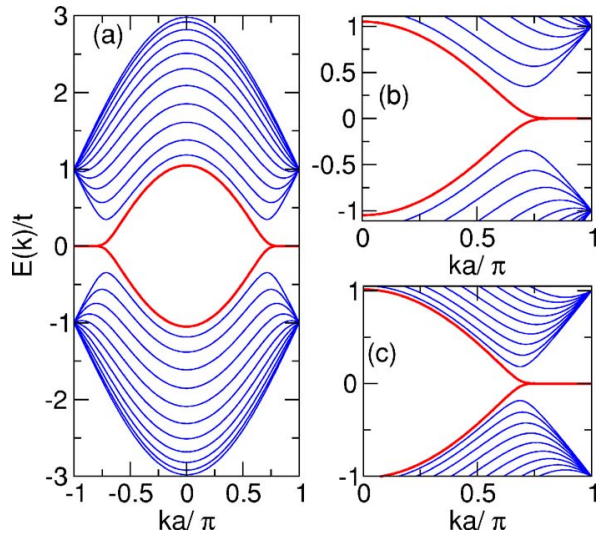

FIG. 2. (Color online) (a) Bands of the ideal zigzag ribbon with 24 atom unit cell. (b) Detail of the low energy sector. (c) Detail of the low energy sector for the 48 atom unit cell

bon is shown in Fig. 3 (lower panel). The honeycomb lattice of $2 \mathrm{D}$ infinite graphene can be generated by a triangular lattice of unit cells with two atoms, labeled $A$ and $B$. Therefore the honeycomb lattice is formed by two interpenetrating sublattices, $A$ and $B$. The first neighbors of atoms in the lattice $A$ belong to sublattice $B$ and vice versa. This underlying structure is responsible for most of the peculiar features of graphene electronic structure. Nanoribbons inherit these properties and, in the case of zigzag nanoribbons, the top and bottom edges belong to atoms on different sublattices. ${ }^{12}$

Figure 2 shows the band structure for the $N=24$ case [Figs. 2(a) and 2(b)] and for the $N=48$ case [only the low energy region is now shown in Fig. 2(c)]. The energy units are given in terms of the hopping parameter $|t| \simeq 3 \mathrm{eV}$ which is the only energy scale in the Hamiltonian. There is a perfect electron-hole symmetry which sets the Fermi energy to zero for half filling. Notice that the density of bands per energy interval is largest for the wider ribbon, as expected. We can distinguish three different regions, (i) $|E|<\Delta_{1}$, (ii) $\Delta_{1}<|E|$ $<|t|$, and (iii) $|E|>t$, where $\Delta_{1}$ is the minimum energy of the second subband closest to $|E|=0$. This energy scale $\Delta_{1}$ is associated to the finite width of the ribbon and it decreases as $N^{-1}$. For the cases considered here, $N=24$ and $N=48, \Delta_{1}$ $=0.35 t$ and $\Delta_{1}=0.18 t$, respectively. In the $N=\infty$ limit $\Delta_{1}$ goes to zero, as expected for two-dimensional graphene.

The density of extra carriers that needs to be injected in the ribbon so that the Fermi energy hits the second subband, $E_{F}=\Delta_{1}$, exceeds the upper experimental limit reached so far, ${ }^{1}$ $\delta n=10^{13} \mathrm{~cm}^{-2}$. Electrical doping up to the second subband could be possible for wider ribbons. Although only the lowest energy region $|E|<\Delta_{1}$ could be accessible experimentally for the narrow ribbons considered here, some insight can be gained also by analyzing the effect of elastic scattering on the transmission at energies in the other two regions. The bands immediately above $\Delta_{1}$ present two positive (and two negative) momenta at a given energy. This results in the doubling of the number of conducting channels at a given energy, as long as $|E|<t$. Higher in energy, $|E|>t$, we find simple parabolic bands which yield one channel per band as in the case of III-V semiconductors.

The energy bands closest to $E_{F}$ are flat for $|k|$ larger than a critical wave vector $k_{c}$, and present dispersion otherwise. 

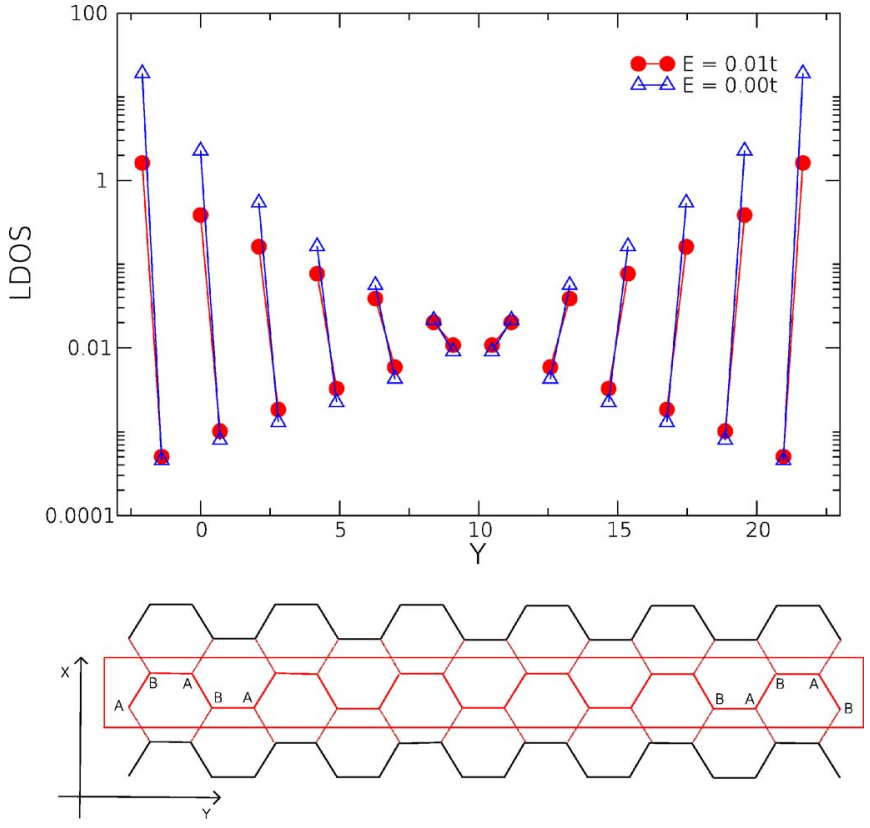

FIG. 3. (Color online) Upper panel: Local density of states of the ideal 24 atoms-wide ribbons for $E=0$ (circles) and $E=0.01 t$ (triangles). Notice the vertical logarithmic scale. Lower panel: atomic structure of a section of a $N=24$ zigzag ribbon.

The cutoff $k_{c}$ is a decreasing function of $N$ which tends to $k_{c} a / \pi=2 / 3$ in the $N=\infty$ limit. ${ }^{9}$ In Fig. 3 we plot the local density of states across a supercell unit for the ribbon with $N=24$ and for two different energies, $E=0$ and $E=0.01 t$. Atoms $A$ and $B$ in the same unit cell are joined by a straight line. In both cases the LDOS is peaked on the edges, although the contrast is larger for the $E=0$ case, which corresponds to the dispersionless states. The LDOS presents a peculiar oscillation, related to the sublattice structure. Starting from the left edge, the LDOS peaks only in $A$, the weight on the first $B$ atom being very small. As we move towards the center the weight on the $A$ atoms decays exponentially whereas the weight on the $B$ atoms increases exponentially. In the center the weight on the $A$ and $B$ atoms is comparable and the weight on the $B$ atoms becomes dominant as we move towards the opposite edge, in very good agreement with the $\vec{k} \cdot \vec{p}$ theory. ${ }^{12}$ As a result, the density of states, disregarding the sublattice index, is peaked at the edges, which permits one to refer to the lowest subband states as "edge states."

It is apparent from Fig. 3 that the $A$ and $B$ edge states become coupled in the middle of the ribbon. Importantly, the structure of the current operator is the same as the hopping part of the Hamiltonian, coupling atoms of the different sublattices. Therefore the current density of a given state, evaluated in a unit cell with two atoms, is proportional to the product of its $A$ and $B$ components, $\psi_{A}(y)$ and $\psi_{B}(y)$. In the continuum limit the current associated to the wave function $\Psi^{\dagger}=\left(\psi_{A}^{*}, \psi_{B}^{*}\right)$ reads $j_{x}(y) \propto\left(\Psi^{\dagger} \sigma_{x} \Psi\right)$, where $\sigma_{x}$ is the Pauli matrix acting on the $A B$ space. ${ }^{5,6,26}$ From Fig. 3 we expect the product $\psi_{A}(y) \times \psi_{B}(y)$ to take similar values in the edges $^{12}$ and in the center of the ribbon. The resulting picture is the following: Whereas the charge density of low energy

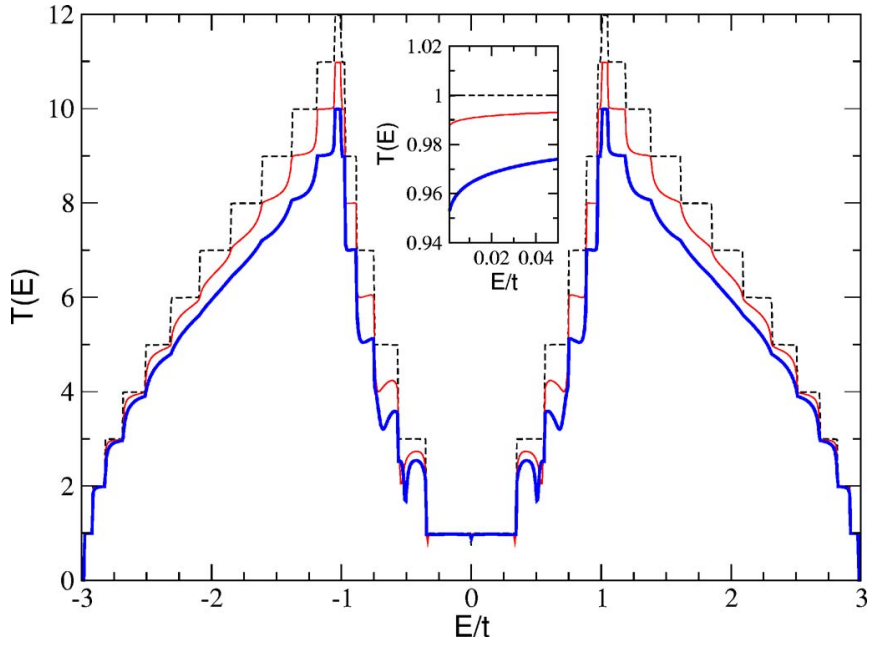

FIG. 4. (Color online) Dashed (black) line: transmission spectrum for ribbons with $N=24$ atoms in the ideal case. Thin (red) line: removing one atom from the edge. Thick (blue) line: removing two atoms on the top and bottom edges. Inset: Detail of the transmission in the low energy sector. The effect of removing one or two atoms on the border is very small in the lowest conductance plateau.

states, proportional to $\rho(y)=\left|\psi_{A}\right|^{2}+\left|\psi_{B}\right|^{2}$, is peaked on the edges, their current density $j_{x}(y)$ is more homogeneously distributed across the ribbon. This picture is substantially different from that of nonrelativistic electrons with scalar wave functions $\phi(x, y)=\psi(y) e^{i k x}$ for which the charge density $\rho(y)=|\psi(y)|^{2}$ and the current density $j_{x}(y) \propto \phi \partial_{x} \phi^{*}-\phi^{*} \partial_{x} \phi$ $\propto k \rho(y)$ have the same profile. The consequences of this difference between the current and charge densities will become apparent later and are one of the results of this work.

\section{B. Defective edges}

We now study the effect of a single vacancy in the edge(s) of the ribbon on the transport properties. From the formal point of view this is done using the approach described in the previous section. The sector of the ribbon where the vacancy is located is treated as the device. In Fig. 4 we plot $T(E)$ for three cases: Ideal case, one atom missing and two atoms missing. As everywhere else in the text, this is the transmission per spin channel. Since we assume that time-reversal symmetry is not broken, the total transmission should be multiplied by a factor of 2 to account for the spin degree of freedom. All of them display electron-hole symmetry around $E=0$. As in the case of the energy bands, the $T(E)$ curves have three different regions. In the large $|E|$ region, the transmission for the ideal ribbon $T_{\text {ideal }}(E)$ is quantized according to the usual law for nonrelativistic electrons and holes: $n e^{2} / h$, where $n$ is the number of bands that intersect the Fermi energy for $k>0$. In this case $n$ goes from 1 to 12 in steps of 1 , consistent with the parabolic dispersion away from the Dirac cones. This large $|E|$ region is not likely to be reached experimentally since it would imply a huge depletion of the charge, but is interesting from the conceptual point of view. In the intermediate region $\left(t<|E|<\Delta_{1}\right)$ $T_{\text {ideal }}(E)$ changes according to the rule $(2 n+1) e^{2} / h$. This re- 


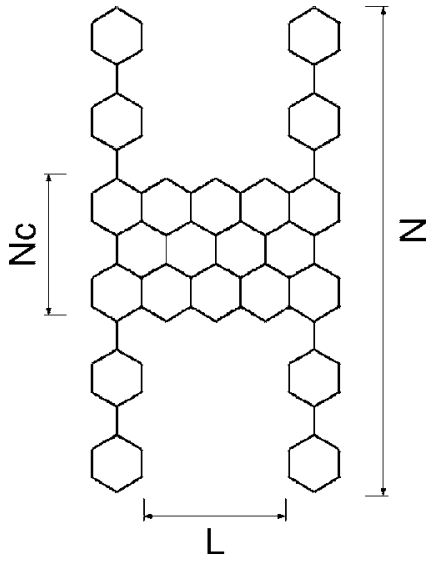

FIG. 5. Structure of the square-shaped nanoconstriction with three characteristic lengths, $N, N_{c}$, and $L$.

sult has been previously obtained by Peres et al. ${ }^{13}$ The factor of two comes from the shape of the bands in this region, as discussed above. The transmission is maximal at the energy where the "Dirac" ladder and the nonrelativistic ladder meet at $E \simeq t$. In the relevant low energy sector, where we will focus our attention from now on, the transmission of the ideal ribbon is one.

The effect of scattering introduced in the ribbons affects very differently the transmission in the different energy regions. The removal of a single atom on the edge erases the $2 n+1$ ladder in the intermediate energy region for the $N$ $=24$ ribbon (see Fig. 4). This points towards a very difficult experimental verification of the $2 n+1$ transmission ladder in these narrow ribbons. In contrast, in the case of $N=48$ the conductance remains quantized in the $2 n+1=3$ plateau after the removal of 1 atom in each edge, but the higher energy plateaus disappear.

In contrast with the higher energy subbands $\left(|E|>\Delta_{1}\right)$, the plateau in the low energy sector is only weakly affected by the removal of atoms in the edges, even for the narrower ribbon $(N=24)$ as can be seen in Fig. 4 . The effect on the transmission in the experimentally relevant low energy sector is below $2 \%$ for the removal of one atom on one edge and below $5 \%$ when one atom is missing on both edges. In contrast, the removal of a single atom in the central part of the ribbon (not shown) has a much larger influence on the low energy transmission. These results are compatible with the fact that the current density carried by edge states is spread along the central region. The robustness of the transmission in the low energy sector and its weakness in the intermediate energy sector are relevant results since atomic size fluctuations in the edges are unavoidable in real samples.

\section{SQUARE CONSTRICTIONS}

We now present results for square-shaped nanoconstrictions as those shown in Fig. 5. We choose this shape because it permits a comparison with a simple model for electrons in parabolic bands. It also seems possible to obtain analytical expressions for the transmission curve for square constrictions using an analytical approach along the lines of previous
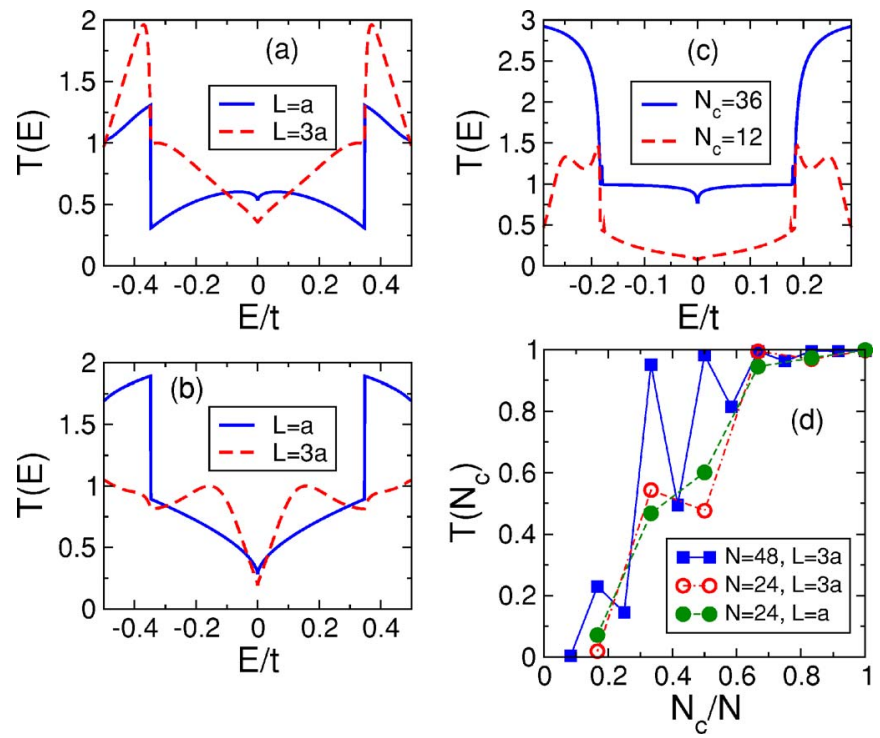

FIG. 6. (Color online) Square constrictions. (a) $T(E)$ curves for $N=24, N_{c}=12$ and two values of $L$. (b) $T(E)$ curves for $N=24, N_{c}$ $=8$ and two values of $L$. (c) $T(E)$ curves for $N=48, L=3 a$ for two constrictions with different $N_{c}$. (d) $T(E=0.05 t)$ as a function of the aspect ratio of the constriction $N_{c} / N$ for three families of constrictions.

work. ${ }^{5,6,12}$ Another feature of square constrictions is the presence of armchair edges joining the zigzag edges of the wide and narrow regions. We consider square constrictions with top-bottom symmetry, like those in Fig. 5, characterized by three lengths: The width of the electrode and the width of the constriction, $N$ and $N_{c}$, both measured in units of the number of atoms of the supercell unit, and the length of the constriction $L$, measured in units of $a$, the graphene lattice parameter.

In Fig. 6 we show transmission curves for a variety of square constrictions. In Figs. 6(a) and 6(b) we show results for electrodes with $N=24$ atoms, whereas in Fig. 6(c) we show results for wider electrodes with $N=48$. The two curves in Fig. 6(a) permit one to compare the transmission for two structures with the same aspect ratio $N_{c} / N=12 / 24$ but different nanoconstriction length $L$. Analogously, Fig. 6(b) shows two $T(E)$ curves with the same aspect ratio $N_{c} / N$ $=8 / 24$ and different $L$. In Fig. 6(c) we show two $T(E)$ curves for a wider electrode, keeping the length of the constriction fixed to $L=3 a$ and changing the aspect ratio. The transmission corresponding to the lowest subband $|E|<\Delta_{1}$ is significantly reduced with respect to the ideal ribbon $T=1$, but remains finite $[T(0) \simeq 0.18]$ even for the narrowest and longest constriction.

In order to highlight the peculiar properties of graphene, we give two arguments, which turn out to be inapplicable, to expect a vanishing transmission at zero energy. First, since low energy transmission is associated to edge states and given that the edges of the electrode and the constriction are not connected, the transmission would vanish as $N_{c}$ becomes much smaller than $N$. This argument fails because the current density of edge states has a sizable contribution in the center of the ribbon, which is smoothly connected to the constrictions considered in Fig. 6. The second argument is based on 
the behavior of a square constriction with parabolic-band electrons. In that case, the different width of the semi-infinite ribbon $W_{r}$ and the constriction $W_{c}$ yields different band minima so that an energy gap is created in the narrow region. This can be modeled with square-shaped barrier of height $V_{0}=\frac{\hbar^{2}}{2 m^{*}}\left(\frac{1}{W_{c}^{2}}-\frac{1}{W_{r}^{2}}\right)$ and length $L, m^{*}$ being the effective mass of the electron. The zero energy transmission of a square barrier is always exponentially vanishing with the length, in contrast to our results. This second argument to expect a vanishing transmission at zero energy also fails because the situation for graphene ribbons is very different: Both wide and narrow sectors of the system have zero energy edge states. In other words, it is not possible to create a gap in the low energy region by changing the width of the system so that it is not possible to create a barrier for the carriers in the lowest energy subband by geometrical means. Interestingly, the transmission through a square potential has been calculated, in the case of an infinitely wide ribbon, and the transmission does not vanish for electrons incident perpendicular to the barrier. ${ }^{27}$

The results in Figs. 6(a) and 6(b) suggest that the low energy transmission is an increasing function of the constriction width $N_{c}$ and fairly insensitive to constriction length $L$. In Fig. 6(d) we plot the transmission at a fixed energy $E$ $=0.05 t$ as a function of $N_{c} / N$ for three families of constrictions. Square symbols correspond to a wide ribbon with $N$ $=48$, and $L=3 a$, whereas closed (open) circle symbols correspond to a narrow ribbon with $N=24$ and $L=a(L=3 a)$. It is apparent that as the aspect ratio increases, the transmission increases in average, with superimposed oscillations. This behavior is different from the analogous curve for transmission as a function of the barrier height $T\left(E, V_{0}\right)$ for parabolicband electrons in the tunneling regime $\left(E<V_{0}\right)$, where the curve does not present oscillations. In the $E>V_{0}$ regime the $T\left(E, V_{0}\right)$ curve can present oscillations. ${ }^{24}$ Therefore the $T\left(N_{c} / N\right)$ curve looks closer to the latter case, even for very small energy. This is related to the lack of a gap in the density of states. It is also interesting that for both wide and narrow electrodes the transmission saturates to 1 for the same value of $N_{c} / N \simeq 0.7$. As a final remark, only in the case of atomically narrow constrictions the transmission becomes almost zero. In summary, square constrictions are not very efficient in blocking electronic transport in zig-zag graphene ribbons.

\section{WEDGE-SHAPED CONSTRICTIONS}

We now turn our attention to a wedge-shaped constriction, shown in Fig. 7. As opposed to the square constrictions considered above, wedge-shaped constrictions are always delimited by zigzag edges. It can also be expected that wedgeshaped constrictions are more stable than square constrictions, although further work should clarify this point. The structures considered have a top-bottom symmetry, in contrast to the asymmetric structures considered in earlier work. ${ }^{21,22}$ Naively, the wedge-shaped constriction would allow for adiabatic transport since the zigzag edge never gets interrupted. In contrast, we see that for ribbons with $N=48$
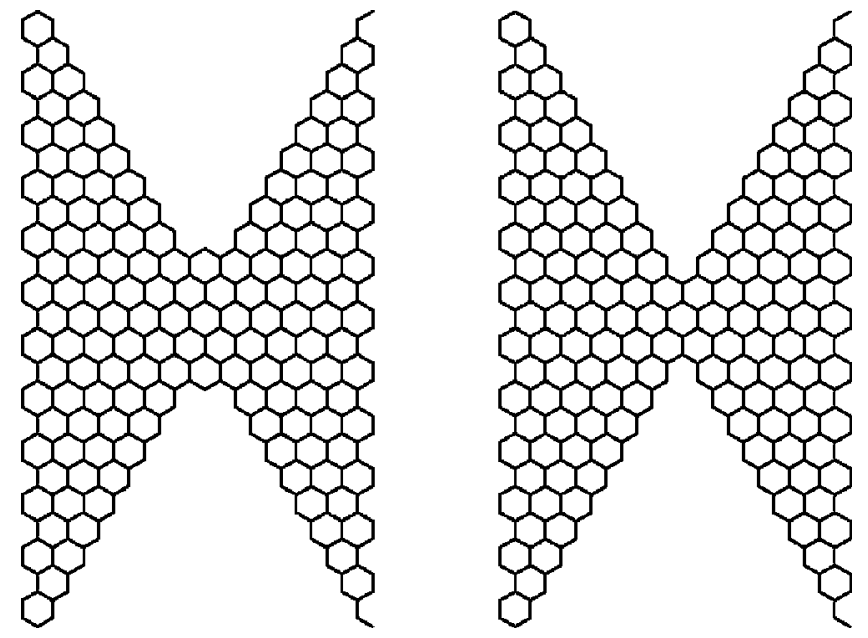

FIG. 7. Schematic atomic structure of wedge-shaped constrictions.

atoms the constrictions shown in Fig. 7 yield a vanishing transmission in the whole low energy sector $|E|<\Delta_{1}$. We have verified that these results are robust against the removal of a single atom from one of the edges. Surprisingly, these smooth nanoconstrictions seem to be more disruptive for low energy transmission than the abrupt nanoconstrictions considered in the previous section.

Interestingly, the vanishing transmission is not associated to a vanishing density of states in the device region, as shown in Figs. 8(c) and 8(d). The peaks in the DOS in the energy region with zero transmission are associated with low energy states very weakly coupled, if at all, to the electrodes. The width of the peaks in the DOS is limited by the numerical broadening used in the calculation. In Fig. 9 we show a contour map of the DOS at $E=0$ and $E=0.04 t$, the energy at which the first peak of the device DOS is located for the structure shown on the right in Fig. 7. The maps reveal lo-
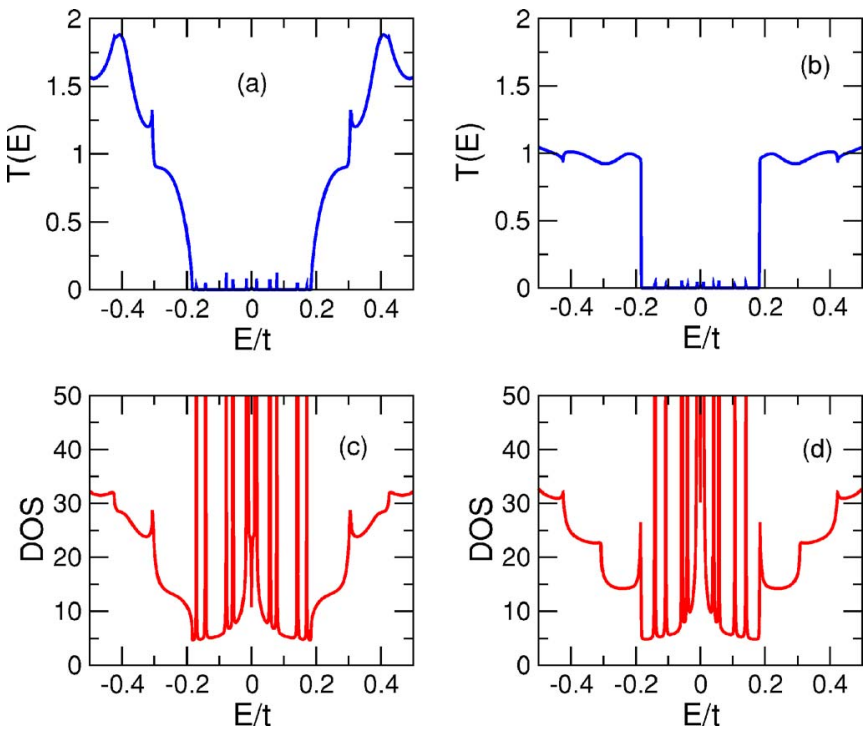

FIG. 8. (Color online) (a) and (b) $T(E)$ for the structures shown in Fig. 7 (blue). (c) and (d) Corresponding density of states projected on the whole constriction region (red). 
(a)
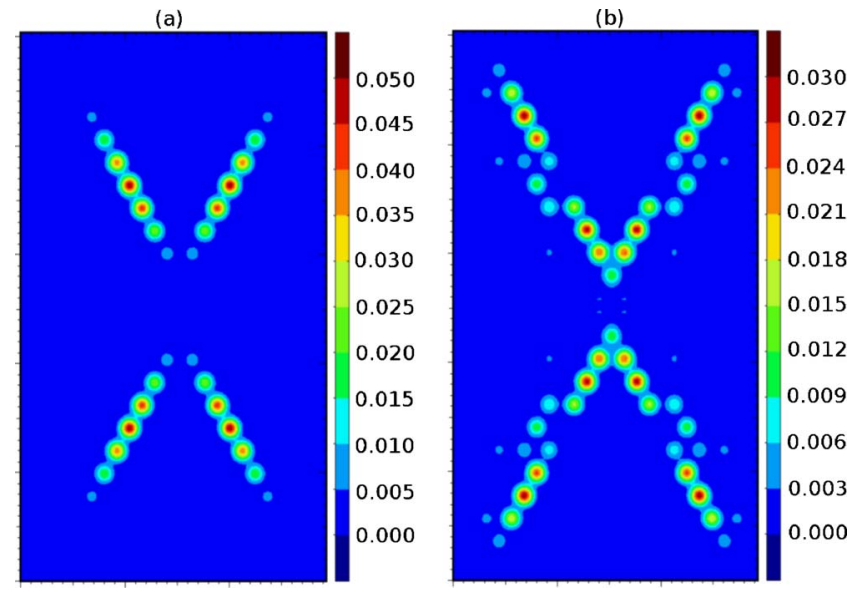

FIG. 9. (Color online) Local density of states for the right wedge structure shown in Fig. 7 at energies $E=0$ (a) and $E=0.04 t$ (b).

calization on the four edges of the constrictions, reflecting the fourfold symmetry of the device. Notice that the LDOS vanishes at the boundary with the electrodes, which explains the vanishing transmission. This LDOS could be observed in STM experiments. ${ }^{19,20}$

Whereas the probability density across one of the four edges has a bell shape for $E=0$, it has a node for the finite energy case. The properties of these low energy nonconducting states in these wedge-shaped structures are very similar to those of zero-dimensional confined states. Confinement in semiconductor heterostructures is associated with the existence of an energy gap. The absence of such a gap in graphene makes it necessary to look for different strategies to confine electrons. ${ }^{27-29}$ In this regard, nanoconstrictions like the ones studied in this section can behave like quantum dots and might permit the study of Coulomb blockade and Kondo physics in graphene structures. The physical origin of these bound symmetric wedge states (SWS) might be related to the formation of Kekule vortex structures discussed in the case of asymmetric wedge states. ${ }^{21,22}$ In the asymmetric case, the suppression of the transmission occurs for very narrow energy windows, since one of the edges is not perturbed. It would be desirable to study whether the vanishing transmission that we have found is related to the vanishing transmission obtained analytically ${ }^{27}$ for the square barrier potential, in infinitely wide ribbons, for incidence angles different from zero. In summary, symmetric wedge constrictions result in a gap in the transmission curve for $|E|<\Delta_{1}$, yet with a finite density of states in that interval, featuring very narrow peaks that mimic a discrete spectrum of confined states.

\section{DISCUSSION AND CONCLUSIONS}

Here we discuss the validity of the approximations assumed for our calculations. Real samples could present several features absent in the idealized ribbon considered here. On one side, the presence of chemical impurities, like hydrogen both in the edge and in bulk, water molecules, oxygen, etc., will affect the electronic structure and transport proper- ties of the system. In a sufficiently clean and ultrahigh vacuum environment the effect of chemical impurities could be negligible. In experiments, the graphene ribbon is deposited on an insulating substrate, which has not been included in our calculation. First-principles calculations indicate that the effect of the substrate is weak. ${ }^{30}$

The use of a single-particle model certainly fails, in a trivial sense, if there are deviations from charge neutrality which would make it necessary to perform a self-consistent calculation including, at least, the Hartree contribution. This is certainly achieved by density functional calculations. We have verified that, for the structures considered here, the electronic density does not deviate significantly from charge neutrality. The single particle approach also fails if the electron liquid happens to be different from a Fermi liquid, in which the low energy quasiparticles have the same quantum numbers than the free electrons. Such a scenario has been considered for two-dimensional graphene. ${ }^{31} \mathrm{~A}$ priori, this is a serious issue in a one-dimensional system, where the Fermi liquid state is not stable with respect to electron-electron interaction and a Luttinger liquid is expected. The same statement applies for nanotubes. However, the single-particle Fermi liquid picture describes most of the experimental results in nanotubes and the same can be expected for graphene ribbons.

We have used the same on-site energy (0) and hopping $t$ for the edge and the inner atoms. This is an approximation both because of the different electric potential felt by the edge and bulk atoms in the ideal lattice and because of the relaxation of the atomic positions of the edge atoms, that we have ignored. Graphene based structures are known to be very stiff and therefore we do not expect important atomic relaxations. In the case of ideal ribbons the use of different tight-binding parameters for inner and edge atoms yields results similar to those obtained with our approximation. ${ }^{11} \mathrm{We}$ have also neglected the nonorthogonality of the atomic basis set. The electronic structure of ideal graphene so obtained is very similar to the $a b$ initio results in an energy window of several hundreds of meV around the Fermi energy. ${ }^{37}$ Since the linear conductance is given by the transmission at the Fermi energy, our transport results are not affected by this approximation. Additionally, ab initio calculations, including all the atomic orbitals, show that the dangling bonds present on the edge atoms form a flatband close to the Fermi energy. ${ }^{23}$ We have also ignored the spin degree of freedom. The flatband at zero energy is expected to spin split due to spontaneous magnetization induced by the Coulomb repulsion. ${ }^{23,32}$ This interesting issue deserves more theoretical and experimental work. We have also ignored spin-orbit interaction, which is very small in carbon, although it has attracted some interest, ${ }^{33,34}$ in part due to the spin Hall effect. $^{35}$

In summary, we have studied coherent transport in graphene nanoribbons with zigzag edges. The electronic structure of the ribbons is described with a simple one orbital tight-binding approximation. We have focused on narrow ribbons $(W<5 \mathrm{~nm})$ for which only the lowest energy subband is expected to be experimentally relevant. Our results could be summarized as follows: (i) The low-energy transport properties are robust with respect to isolated vacancies 
on the edges. (ii) These are also robust with respect to square-shaped nanoconstrictions. Linear conductance survives in most cases except for very narrow constrictions. This is at odds with the behavior of parabolic-band electrons in similar constrictions and reflects two nontrivial features of edge states: Their minimum energy is independent of the ribbon width and their current density profile spreads beyond the edges of the ribbon, in contrast with their density profile. (iii) In contrast to square constrictions, wedge-shaped nanoconstrictions result in a gap in the transmission and result in a zero linear conductance which is related to the appearance of localized low energy edge states. These edge states have properties similar to those of confined states in zero dimensions. Therefore graphene wedge shaped constrictions might have properties analogous to those of semiconductor quan- tum dots. Extensions of this work will address the spin degree of freedom, topological defects, ${ }^{36}$ and armchair edges.

Note added. After the completion of this work we became aware of related theory papers ${ }^{38,39}$ with ballistic transport calculations similar to ours.

\section{ACKNOWLEDGMENTS}

We acknowledge useful discussions with M. Vozmediano, A. Cortijo, and B. Valenzuela. This work has been financially supported by MEC-Spain (Grants No. FIS200402356, No. MAT2005-07369-C03-01, and the Ramon y Cajal Program) and by Generalitat Valenciana (GV05-152). This work has been partly funded by FEDER funds.
*Electronic address: jfrossier@ua.es

${ }^{1}$ J. S. Bunch, Y. Yaish, M. Brink, K. Bolotin, and P. L. MacEuen, Nano Lett. 5, 287 (2005).

${ }^{2}$ K. S. Novoselov, A. K. Geim, S. V. Morozov, D. Jiang, M. I. Katsnelson, I. V. Grigorieva, S. V. Dubonos, and A. A. Firsov, Nature (London) 438, 197 (2005).

${ }^{3}$ Y. Zhang, Y. W. Tan, H. L. Stormer, and P. Kim, Nature (London) 438, 201 (2005).

${ }^{4}$ C. Berger, Z. Song, X. Li, X. Wu, N. Brown, C. Naud, D. Mayou, T. Li, J. Haas, A. N. Marchenkov, E. H. Conrad, P. N. First, and W. A. de Heer, Science 312, 1191 (2006).

${ }^{5}$ M. I. Katsnelson, Eur. Phys. J. B 51, 157 (2006).

${ }^{6}$ J. Tworzydlo, B. Trauzettel, M. Titov, A. Rycerz, and C. W. J. Beenakker, Phys. Rev. Lett. 96, 246802 (2006).

${ }^{7}$ K. Nomura and A. H. MacDonald, cond-mat/0606589 (unpublished).

${ }^{8}$ G. W. Semenoff, Phys. Rev. Lett. 53, 2449 (1984).

${ }^{9}$ K. Nakada, M. Fujita, G. Dresselhaus, and M. S. Dresselhaus, Phys. Rev. B 54, 17954 (1996).

${ }^{10}$ K. Wakabayashi, M. Fujita, H. Ajiki, and M. Sigrist, Phys. Rev. B 59, 8271 (1999).

${ }^{11}$ M. Ezawa, Phys. Rev. B 73, 045432 (2006).

${ }^{12}$ L. Brey and H. A. Fertig, Phys. Rev. B 73, 235411 (2006).

${ }^{13}$ N. M. R. Peres, A. H. Castro Neto, and F. Guinea, Phys. Rev. B 73, 195411 (2006); 73, 239902(E) (2006).

${ }^{14}$ R. Saito, M. S. Dresselhaus, and G. Dresselhaus, Physical Properties of Carbon Nanotubes (Imperial College Press, London, 1998).

${ }^{15}$ A. Javey, J. Guo, Q. Wang, M. Lundstrom, and H. Dai, Nature (London) 424, 654 (2003).

${ }^{16}$ P. Jarillo-Herrero, S. Sapmaz, C. Dekker, L. P. Kouwenhoven, and H. van der Zant, Nature (London) 429, 389 (2004).

${ }^{17}$ J. Nygard, D. H. Cobden, and P. E. Lindelof, Nature (London) 408, 342 (2000).

${ }^{18}$ P. Jarillo-Herrero, J. Kong, H. van der Zant, C. Dekker, L. P. Kouwenhoven, and S. De Franceschi, Nature (London) 434, 484 (2005).

${ }^{19}$ Y. Niimi, T. Matsui, H. Kambara, K. Tagami, M. Tsukada, and H. Fukuyama, Appl. Surf. Sci. 241, 43 (2005).

${ }^{20}$ Y. Kobayashi, K. I. Fukui, T. Enoki, K. Kusakabe, and Y.
Kaburagi, Phys. Rev. B 71, 193406 (2005).

${ }^{21}$ K. Wakabayashi and M. Sigrist, Phys. Rev. Lett. 84, 3390 (2000).

${ }^{22}$ K. Wakabayashi, Phys. Rev. B 64, 125428 (2001).

${ }^{23}$ Y. Miyamoto, K. Nakada, and M. Fujita, Phys. Rev. B 59, 9858 (1999); H. Lee, N. Park, Y.-W. Son, S. Han, and J. Yu, Chem. Phys. Lett. 398, 207 (2004); T. Kawai, Y. Miyamoto, O. Sugino, and Y. Koga, Phys. Rev. B 62, R16349 (2000).

${ }^{24}$ S. Datta, Electronic Transport in Mesoscopic Systems (Cambridge University Press, Cambridge, England, 1995).

${ }^{25}$ D. Gunlycke, H. M. Lawler, and C. T. White, cond-mat/0606693 (unpublished).

${ }^{26}$ V. V. Cheianov and V. I. Fal'ko, Phys. Rev. B 74, 041403(R) (2006).

${ }^{27}$ M. I. Katsnelson, K. S. Novoselov, and A. K. Geim, Nat. Phys. 2, 620 (2006).

${ }^{28}$ P. G. Silvestrov and K. B. Efetov, cond-mat/0606620 (unpublished).

${ }^{29}$ J. M. Pereira, Jr., V. Mlinar, F. M. Peeters, and P. Vasilopoulos, Phys. Rev. B 74, 045424 (2006).

${ }^{30}$ B. Obradovic, R. Kotlyar, F. Heinz, P. Matagne, T. Rakshit, M. D. Giles, M. A. Stettler, and D. E. Nikonov, Appl. Phys. Lett. 88, 142102 (2006).

${ }^{31}$ J. González, F. Guinea, and M. A. H. Vozmediano, Phys. Rev. Lett. 77, 3589 (1996).

${ }^{32}$ M. A. H. Vozmediano, M. P. Lopez-Sancho, T. Stauber, and F. Guinea, Phys. Rev. B 72, 155121 (2005); N. M. R. Peres, F. Guinea, and A. H. Castro, Phys. Rev. B 72, 174406 (2005).

${ }^{33}$ L. Chico, M. P. López-Sancho, and M. C. Muñoz, Phys. Rev. Lett. 93, 176402 (2004).

${ }^{34}$ D. Huertas-Hernando, F. Guinea, and A. Brataas, cond-mat/ 0606580 (unpublished).

${ }^{35}$ C. L. Kane and E. J. Mele, Phys. Rev. Lett. 95, 226801 (2005).

${ }^{36}$ A. Cortijo and M. A. H. Vozmediano, cond-mat/0603717 (unpublished).

${ }^{37}$ S. Reich, J. Maultzsch, C. Thomsen, and P. Ordejón, Phys. Rev. B 66, 035412 (2002).

${ }^{38}$ A. Rycerz, J. Tworzydlo, and C. W. J. Beenakker, cond-mat/ 0608533 (unpublished).

${ }^{39}$ T. C. Li and S. P. Lu, cond-mat/0609009 (unpublished). 